\title{
APERTURE SYNTHESIS OBSERVATIONS OF M17 SW
}

\author{
Y. WANG, S. ZHOU, N. EVANS II, D. JAFFE \\ University of Texas at Austin, Austin, TX 78712, USA
}

\author{
M. HAYASHI \\ University of Tokyo, Bunkyo-ku, Tokyo 113, Japan
}

Y. MURATA

ISAS, Sagamihara Kanagawa 229, Japan

\begin{abstract}
We present maps of M17 SW cloud core in the CS J=1 $\rightarrow 0$ line and the $49 \mathrm{GHz}$ continuum obtained with the Nobeyama Millimeter Array.
\end{abstract}

The M17 SW molecular cloud core lies just southwest of the optical HII region, M17 (NGC 6618) at a distance of $\sim 2.2 \mathrm{kpc}$. High resolution radio continuum maps (Felli, et al., 1984) indicate an almost edge-on geometry of the interface between the dense molecular core and the HII region, making M17 SW one of the best regions for studying the interaction between an HII region and a molecular cloud and the resultant photodissociation region. Recent high resolution observations of the region have directly shown the clumpy nature of the cloud core. Stutzki and Güsten (1990) suggest that M17 SW contains 180 clumps based on their $13^{\prime \prime}$ resolution $\mathrm{C}^{18} \mathrm{O} \mathrm{J}=2 \rightarrow 1$ observations. Our multitransition $\mathrm{CS}$ and $\mathrm{C}^{34} \mathrm{~S}$ observations reveal the clumpy structure of the dense gas on scales of about $0.2 \mathrm{pc}\left(20^{\prime \prime}\right)$. The detailed excitation analyses of the multitransition data shows a fairly uniform, high density $\left(6 \times 10^{5} \mathrm{~cm}^{-3}\right)$ throughout the entire core (Wang, et al., in preparation). Prominent condensations seen in the CS $\left(\mathrm{C}^{34} \mathrm{~S}\right)$ line temperature maps do not show up as density enhancements, but rather as column density peaks. Along with other evidence, these results imply a cloud model in which the CS emission arises from many dense clumps, still smaller than our $20^{\prime \prime}$ resolution.

In order to test the clumpy cloud picture and to reveal smaller scale structure of the dense gas, we observed the $\mathrm{HII} / \mathrm{H}_{2}$ interface with the Nobeyama Millimeter Array in the $\mathrm{CS} \mathrm{J}=1 \rightarrow 0$ line and in the $49 \mathrm{GHz}$ continuum. Two fields were observed toward the interface which covers an area of $2.5^{\prime} \times 4^{\prime}$. The synthesized beam was $10^{\prime \prime} \times 8^{\prime \prime}$ for the northern field and $12^{\prime \prime} \times 9^{\prime \prime}$ for the southern field. We combined the two fields using the Maximum Entropy Method, convolving with a circular beam of $10^{\prime \prime} \times 10^{\prime \prime}$.

Figure 1 shows the maps of the $49 \mathrm{GHz}$ continuum and the velocity integrated CS $\mathrm{J}=1 \rightarrow 0$ line. The continuum map clearly shows an arc-shaped emission region surrounding the ultracompact HII region, depicting the location of the ionization front (Felli, et al., 1984). A weaker emission feature is found to the south of the emission arc. 

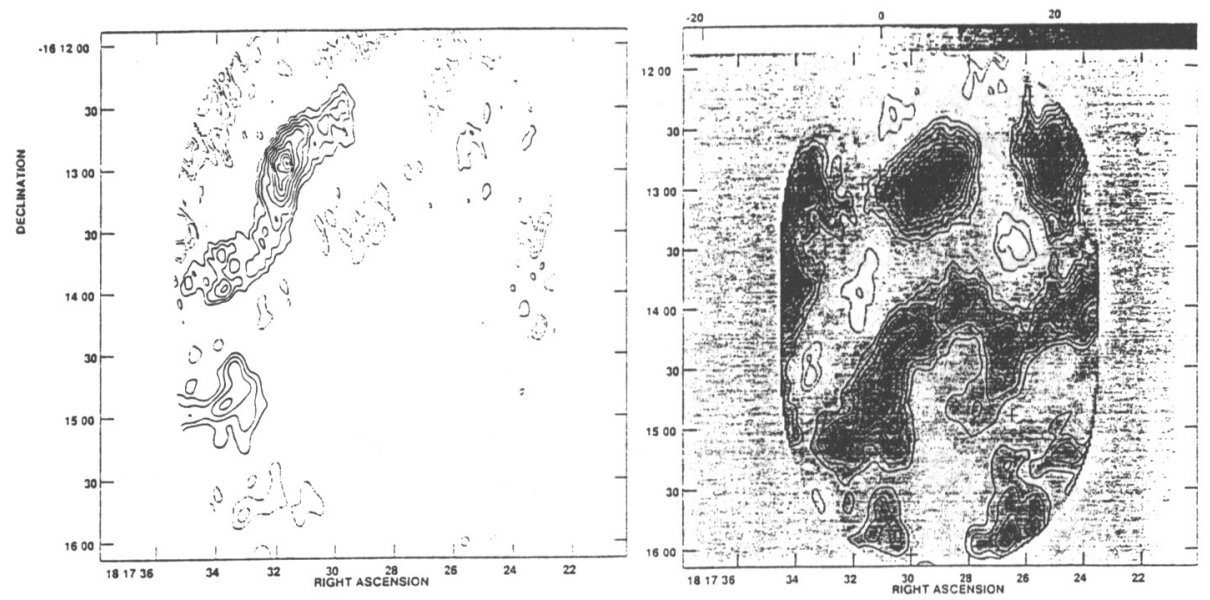

FIGURE I Left panel is the $49 \mathrm{GHz}$ continuum map. Contour levels are $-6 \sigma,-4 \sigma, 4 \sigma, 6 \sigma, \ldots . ., 22 \sigma$. Right panel is the velocity integrated CS J $=1 \rightarrow 0$ map. Contour levels are $-2 \sigma, 2 \sigma, \ldots . ., 10 \sigma$.

The velocity integrated $C S \mathrm{~J}=1 \rightarrow 0$ map has excellent correspondence with the single-dish CS maps. Features seen in the single-dish $\mathrm{CS}$ and $\mathrm{C}^{34} \mathrm{~S}$ maps become more prominent and distinctive in the interferometric map. About 15 clumps are identifiable in the velocity integrated map and the velocity channel maps. Individual clumps have sizes of $\sim 20^{\prime \prime}-60^{\prime \prime}$ and linewidths of $1-2 \mathrm{~km} \mathrm{~s}^{-1}$. We compared the CS $J=1 \rightarrow 0$ channel maps with the clumps deconvolved from the $13^{\prime \prime}$ resolution $\mathrm{C}^{18} \mathrm{O} \mathrm{J}=2 \rightarrow 1$ maps (Stutzki and Güsten 1990) and found that only the most prominent clumps which dominate the overall single dish CS emission show up clearly in the $10^{\prime \prime}$ resolution CS $\mathrm{J}=1 \rightarrow 0$ maps. Further analysis and comparison of the data with the results of the single dish multitransition CS and $\mathrm{C}^{34} \mathrm{~S}$ studies remain to be carried out to be able to understand the density structure of these clumps.

\section{REFERENCES}

Felli, M., Churchwell, E., and Massi, M.: 1988, A\&A, 136, 53

Stutzki, J. and Güsten, R.: 1990, ApJ, 356, 513 